\title{
HISTÓRICO DAS AÇÕES DE CONTROLE DA FILARIOSE LINFÁTICA EM OLINDA, PERNAMBUCO, BRASIL
}

Abraham Rocha ${ }^{1,2}$, Elizabete Miguel dos Santos ${ }^{3}$, Paula Oliveira ${ }^{1}$ e Eduardo Brandão $^{1}$

\section{RESUMO}

A filariose linfática é uma parasitose que afeta regiões tropicais e subtropicais. No Brasil, apenas a região metropolitana de Recife (Recife, Olinda, Jaboatão dos Guararapes e Paulista) ainda é considerada como foco ativo de transmissão da parasitose. Diante disso, o objetivo deste trabalho foi descrever o histórico das atividades de controle da parasitose em Olinda$\mathrm{PE}$, área que por muito tempo apresentou número significativo de casos, evidenciando as principais ações desenvolvidas desde o ano de 1987. Ao longo de aproximadamente 30 anos, muitos estudos clínico-laboratoriais e epidemiológicos foram desenvolvidos no município. Recentemente, mais de cinco ciclos de tratamento em massa com citrato de dietilcarbamazina foram realizados na área, com uma adesão de mais de $65 \%$ de toda a população, o que contribuiu para a redução da microfilaremia local a taxas inferiores a $1 \%$. Esse fato viabilizou a implantação da pesquisa de avaliação da transmissão, etapa decisiva para definição da situação atual da filariose linfática e de novas estratégias de controle e vigilância.

DESCRITORES: Wuchereria bancrofti; filariose; Olinda; programa de controle.

\section{ABSTRACT}

History of lymphatic filariasis control actions in Olinda, Pernambuco, Brazil.

Lymphatic filariasis is a parasitic disease that affects tropical and subtropical regions. In Brazil, only the metropolitan area of Recife (Recife, Olinda, Jaboatão dos Guararapes and Paulista) has been considered an active transmission focus of the parasite. The purpose of this study was to describe the history of the control activities of this parasitic disease, showing the main actions developed since 1987 in Olinda-PE, an area that has long presented a significant number of cases. Over the last 30 years, many clinical, laboratorial and epidemiological studies have been developed in this city. Recently, more than five mass treatment cycles with diethylcarbamazine

\footnotetext{
1. Serviço de Referência Nacional em Filarioses, Departamento de Parasitologia, Centro de Pesquisas Aggeu Magalhães, Fundação Oswaldo Cruz, Recife, PE, Brasil.

2. Laboratório do Hospital Otávio de Freitas, Secretaria de Saúde do Estado de Pernambuco, Brasil

3. Pós Graduação em Saúde Coletiva, Faculdade Redentor, Recife-PE, Brasil.
}

Endereço para correspondência: Abraham Rocha. Departamento de Parasitologia, Serviço de Referência Nacional em Filarioses, Av. Moraes Rego S/N, CEP 50670-420, Recife-PE, Brasil. E-mail: rocha@cpqam.fiocruz.br 
citrate were performed in the area, with the support of over $65 \%$ of the population, which contributed to a reduction of the local microfilaremia to rates under $1 \%$. This fact enabled transmission assessment surveys, a decisive step to define the current situation of LF and new control and surveillance strategies

KEY WORDS: Wuchereria bancrofti; filariasis; Olinda; control programme.

\section{INTRODUÇÃO}

A filariose linfática (FL), também conhecida como elefantíase, é uma enfermidade parasitária que apresenta um amplo espectro de manifestações clínicas e que há mais de 20 anos vem ocupando o segundo lugar no ranking mundial das doenças incapacitantes (WHO, 2015). De acordo com dados da Organização Mundial da Saúde (OMS), esta parasitose ocorre em 73 países, sendo estimado em cerca de 1,4 bilhão o número de indivíduos que vivem em áreas de risco de adquirir a infecção e em 120 milhões o número de infectados (108 milhões por Wuchereria bancrofti e 12 milhões por Brugia malayi e Brugia timori) (OMS, 2015). Nas Américas, onde só ocorre a infecção por $W$. bancrofti, aproximadamente 11 milhões de pessoas estão sob o risco de adquirir a doença e 720 mil estão infectadas em países com registro de transmissão ativa como República Dominicana, Guiana, Brasil e Haiti, sendo este último responsável pela maior parte dos casos (OPAS, 2007).

Como o agente etiológico desta parasitose não possui reservatórios em potencial, a OMS, em assembleia realizada em 1997, conclamou os Estados Membros para a adoção do Plano Global de Eliminação da Filariose Linfática (PGEFL), por meio da Resolução 50.29, tendo por fim a eliminação desta endemia até 2020 (Molyneux et al., 2000). As principais estratégias do PGEFL estão sedimentadas em dois importantes pilares: 1) interrupção da transmissão por meio da implantação do tratamento em massa (TM) com citrato de dietilcarbamazina (DEC), isolado ou associado ao albendazol ou à ivermectina em dose única anual; 2) prevenção e alívio do sofrimento dos portadores da morbidade (Ottesen et al., 1997).

No Brasil, já foi extinta a maior parte das áreas endêmicas existentes na década de 1950 (MS, 1985). Inquéritos realizados ao longo dos últimos anos não detectaram casos da parasitose nas cidades de Belém (PA) e Maceió (AL), que estavam entre os focos de maior expressão do ponto de vista epidemiológico, o que indica a eliminação da transmissão local e o controle da infecção (Rocha et al., 2005; Freitas et al., 2008; Fontes et al., 2012). Atualmente, quatro municípios da região metropolitana de Recife (RMR), em Pernambuco, constituem o único foco remanescente de FL no País: Recife, Jaboatão dos Guararapes, Paulista e Olinda (MS, 2012).

Em Olinda, o TM foi iniciado em 2005, no bairro Alto do Sol Nascente, área eleita por ter a prevalência de microfilarêmicos maior que 
$1 \%$ e também por ser de elevado risco socioambiental. Neste município, foi adotada a estratégia de tratamento "porta a porta" supervisionada, ao contrário do ocorrido em Recife, onde o tratamento foi realizado por meio de demanda espontânea, estimulada por campanhas de mobilização comunitária (Rocha et al., 2010).

Após a verificação da quebra de transmissão com vistas à eliminação prevista para 2020, os países endêmicos deverão construir um dossiê e submetê-lo à OMS, congregando todas as ações realizadas ao longo dos anos pelos Planos Nacionais de Eliminação da FL (PNEFL) para a obtenção da certificação de controle e eliminação da parasitose (PAHO, 2015). Decorridas aproximadamente duas décadas desde a implantação do PNEFL do Brasil, uma gama de conhecimentos foi produzida e avanços foram alcançados, os quais resultaram em uma redução significativa da prevalência da FL no País. Diante disso, a presente série histórica teve como objetivo consolidar as informações obtidas sobre o agravo no município de Olinda-PE e, dessa maneira, contribuir para a construção do dossiê de eliminação da FL no Brasil, destacando os estudos realizados desde a identificação do foco até as ações de controle com vistas à eliminação.

\section{METODOLOGIA}

\section{Área de estudo}

O município de Olinda, situado no estado de Pernambuco, Brasil, está localizado na RMR e possui uma população de 375.559 habitantes. Localiza-se a $6 \mathrm{~km}$ de Recife, capital pernambucana, e faz limite ao norte com o município de Paulista, ao sul e oeste com Recife e a este com o Oceano Atlântico (IBGE, 2010).

\section{Tipo de estudo e coleta dos dados}

O estudo consiste em uma revisão sistemática do histórico da FL no município de Olinda. Para isso, foi realizada uma pesquisa no banco de dados da biblioteca do Centro de Pesquisas Aggeu Magalhães (CPqAM)/Fundação Oswaldo Cruz (FIOCRUZ). Foram pesquisados manuais, anais de congresso, monografias, dissertações e teses que abordassem o tema Filariose em Olinda, tendo sido feito também o levantamento de dados nas principais bases on-line (biblioteca virtual eletrônica - Bireme, Scielo, Lilacs e PubMed).

As buscas de dados foram conduzidas pelas palavras-chave, em português ou em inglês, filariose, filariose linfática ou Wuchereria bancrofti, no período de agosto de 1987 a dezembro de 2015. Somaram-se a esses descritores as seguintes palavras: diagnóstico, tratamento coletivo, programa 
de controle e eliminação, epidemiologia, morbidade e dietilcarbamazina, sempre combinadas com a especificação município de Olinda ou Olinda.

A leitura analítica, a estratificação e a análise dos dados foram realizadas no período de outubro de 2014 a janeiro de 2016.

\section{RESULTADOS E DISCUSSÃO}

\section{Breve histórico da FL no Brasil}

Inquéritos hemoscópicos realizados no período de 1951 a 1958 permitiram obter dados básicos sobre a prevalência da FL em nosso país e a identificação de focos autóctones em 11 cidades: Ponta Grossa (SC), 14,5\%; Belém (PA), 9,8\%; Barra de Laguna (SC), 9,4\%; Recife (PE), 6,9\%; Castro Alves (BA), 5,9\%; Florianópolis (SC), 1,4\%; São Luís (MA), 0,6\%; Salvador (BA), 0,4\%; Maceió (AL), 0,3\%; Manaus (AM), 0,2\% e Porto Alegre (RS), 0,1\% (Rachou, 1960; MS, 1985). Em decorrência de elevadas prevalências, densidade demográfica e população vetorial, as cidades de Recife-PE e BelémPA foram eleitas pelo Programa de Combate à FL como áreas prioritárias (MS, 1985). Com a identificação dos casos positivos nestas duas áreas e o consequente tratamento seletivo com a DEC, os índices de microfilarêmicos diminuíram drasticamente em Recife-PE, de 6,9\% (1954) para 1,5\% (em 1983), e Belém-PA, de 9,4\% (em 1957) para 0,2\% (em 1983) (FUNASA, 1997).

Com o intuito de avaliar a real situação da FL em Recife, em maio de 1986, no Departamento de Parasitologia do CPqAM/FIOCRUZ, criouse o Grupo de Pesquisa em Filariose, com a realização de atendimento clínico, diagnóstico e avaliação vetorial (Coutinho et al., 1996). No período de 1986-1987, foram avaliados 900 indivíduos dos diversos bairros da região metropolitana de Recife, dos quais 252 (28\%) foram diagnosticados microfilarêmicos e/ou apresentavam alguma forma clínica da doença. Além de evidenciar a FL como um problema de saúde pública, esse inquérito demonstrou, pela primeira vez, que a parasitose estava em franca expansão, sendo notificados casos autóctones dos municípios de Jaboatão dos Guararapes e Olinda, áreas nas quais até então não haviam sido notificados casos de FL ao Ministério da Saúde (MS, 1985; Dreyer, 1987).

A identificação do foco de Olinda, no ano de 1986 (Dreyer e Rangel, 1987), representou um marco no estabelecimento de estratégias de prevenção e controle da filariose neste município. As etapas seguintes estão cronologicamente descritas a seguir. 
Durante esse período, vários inquéritos epidemiológicos foram realizados no município de Olinda envolvendo o CPqAM e a Secretaria de Saúde de Olinda. Em um desses inquéritos, realizado por meio do exame de gota espessa de sangue, foram examinados 751 indivíduos oriundos de três bairros (Triângulo de Peixinhos, Bultrins e Ilha de Santana), sendo identificados casos autóctones de FL e prevalência de microfilarêmicos que variou de 5\% a 10,3\% (Coutinho et al., 1996). Esses dados foram apresentados no I Simpósio Nacional de Filariose, realizado no período de 15 a 18 de setembro de 1987.

Em 1992, Medeiros e colaboradores desenvolveram estudos com indivíduos microfilarêmicos autóctones procedentes das três áreas endêmicas da RMR (Recife, Olinda e Jaboatão dos Guararapes) com o objetivo de investigar a densidade de microfilárias e o índice de infectividade dos vetores (presença de larvas L3). Os autores verificaram que, em Jaboatão dos Guararapes e Olinda, cerca de $65,6 \%$ dos indivíduos microfilarêmicos apresentaram elevadas cargas parasitárias ( $>500 \mathrm{Mf} / \mathrm{mL}$ ), ao contrário de Recife que apresentou densidade inferior a $500 \mathrm{Mf} / \mathrm{mL}$. Foram obtidos, nas três áreas investigadas, em média 35 mosquitos por aspiração em cada casa para a avaliação da taxa de infectividade. Olinda apresentou índices duas vezes maiores do que os encontrados em Recife. Em razão de elevada prevalência, densidade parasitária e infectividade dos vetores, os autores, já naquela época, sugeriram o TM como medida para bloquear a expansão da FL nas áreas endêmicas.

Estudos realizados por Maciel e colaboradores (1994), em Sapucaia e Salgadinho (Olinda), Santo Amaro e Campo Grande (Recife), identificaram prevalências similares nos bairros dos dois municípios (Olinda 12,3\% e Recife 13,5\%). Contudo, os indivíduos residentes em Olinda apresentaram densidade de microfilárias 1,75 vez maior do que os residentes em Recife. Além disso, os estudos entomológicos demonstraram elevados índices $(1,2 \%)$ de infectividade vetorial, particularmente nos bairros de Peixinhos e Sapucaia de Fora, onde cada mosquito possuía de 1 a 14 larvas infectantes, com média de 5 larvas por inseto.

\section{Período de 1998 a 2008}

Em 2001, Braga e colaboradores avaliaram os indicadores socioambientais envolvidos no risco de a população adquirir a FL em Olinda. Dentre eles, destacou-se a rede geral de esgotamento sanitário. Esse estudo possibilitou, pela primeira vez, a classificação das áreas endêmicas em relação à transmissão da FL em três estratos: 1) baixo risco; 2) médio risco e 3) alto risco. Dos 42 casos positivos diagnosticados no estudo, $36(85,7 \%)$ eram oriundos das áreas que apresentaram elevado risco de transmissão. Além dessa informação, os autores também identificaram que quanto maior era a 
proximidade das residências com as fontes hídricas (rios, canais e córregos), maior era o risco de transmissão da FL, uma vez que estes são ambientes propícios para criadouros do vetor.

Inquéritos realizados na comunidade de Azeitona, bairro de Peixinhos em Olinda, identificaram, por meio do exame parasitológico da gota espessa, 87 (11\%) indivíduos microfilarêmicos de um total de 792 investigados (Miranda et al., 2005). Braga e colaboradores (2005), investigando o comportamento da infecção filarial durante a puberdade, estudaram 1.511 jovens oriundos de Olinda, de ambos os sexos, na faixa etária de 9 a 16 anos. Esse estudo evidenciou que a prevalência da FL estava diretamente relacionada com o aumento da idade e que a densidade da carga parasitária no sexo masculino foi 3,6 vezes maior do que no feminino. Por outro lado, a avaliação por meio dos testes de antígeno circulante filarial demonstrou que não havia diferença significativa entre sexo, idade e prevalência de microfilaremia.

Após a realização de um extenso trabalho de identificação e tratamento dos indivíduos microfilarêmicos ao longo dos anos, nos diversos bairros em Olinda-PE, a Secretaria Municipal de Saúde (SMS) identificou a necessidade de reavaliar estes bairros para verificar o impacto das ações desenvolvidas e definir as próximas etapas. Foram então selecionados os bairros Alto da Bondade e Alto da Conquista para a reavaliação da prevalência, utilizando-se a gota espessa (arrastão). Foram investigados mais de 50\% dos residentes em ambas as áreas, obtendo-se uma prevalência de microfilaremia de, aproximadamente, $0,70 \%$, o que não evidenciou a necessidade de implantação do TM. Ainda assim, a SMS levantou a hipótese da necessidade de uma intervenção massiva (Rocha et al., 2010).

Diante do impasse de se adotar ou não o TM nas áreas reavaliadas - Alto da Bondade e Alto da Conquista - em virtude dos dados obtidos no arrastão, a partir de março de 2007 foi desenvolvido um projeto de pesquisa com o objetivo de avaliar tal necessidade (Rocha et al., 2010). Nesse estudo foram envolvidas quatro escolas com crianças em idade de 5 a 18 anos. O teste rápido do cartão ICT foi utilizado para avaliar a presença do antígeno circulante filarial (ACF). Das 672 crianças avaliadas, 66 revelaram-se positivas, ficando assim demonstradas a prevalência entre os escolares de $9,8 \%$ e a necessidade de implementação do TM nas respectivas áreas (Brandão et al., 2007).

Período de 2009 a 2015

Com o objetivo de produzir conhecimento no campo da morbidade filarial (MoF), Marcondes (2010), pela primeira vez, estudou os bairros Alto da Conquista e Alto da Bondade, em Olinda, investigando o conhecimento e as práticas dos portadores da MoF. O estudo envolveu 373 deles no período de 2007 a 2010. Os resultados revelaram que os portadores da MoF possuíam um bom conhecimento sobre a transmissão da doença, porém poucos sabiam 
sobre como se realizava o diagnóstico. Com relação ao tratamento, 59,5\% não sabiam como tratar a doença e, sobre a cura, 68,4\% demonstraram ter conhecimento adequado. Quanto à profilaxia, a maioria desses indivíduos declarou evitar a picada do mosquito mediante o uso de mosquiteiros. Durante a realização desse inquérito, foi verificada a necessidade de maior incentivo e divulgação das práticas de cuidado com o lixo e entulhos. Dessa forma, a autora concluiu que, nas áreas endêmicas investigadas, vigoram diferentes concepções e saberes acerca da FL, inferindo que essas diferenças precisam ser mais bem estudadas pelos serviços de saúde de modo que se alcance mais sucesso nas ações de controle e eliminação da doença no município.

Em 2012, Cabral realizou um trabalho pioneiro no qual estudou a população não aderente ao TM para FL em Olinda. Ela avaliou 102 indivíduos, identificando um percentual de positividade do ACF através do teste do cartão ICT de 3,92\%. No entanto, esses indivíduos ACF-positivos não apresentaram microfilaremia quando avaliados pelo padrão-ouro parasitológico da filtração de sangue noturno em membrana de policarbonato (FSNM). O estudo revelou que os principais motivos para a não adesão dos residentes ao TM foram o não recebimento da medicação e as reações adversas produzidas pelo medicamento. Esses resultados chamam a atenção para a necessidade do estabelecimento de novas estratégias para aumentar a adesão da população ao tratamento, principalmente no que se refere ao risco-benefício do uso da medicação.

Ainda nesse período, Silva (2014) avaliou a quebra da transmissão da FL após a expansão do TM. Nesse estudo, foram avaliadas 337 crianças, entre 5 e 18 anos, residentes no bairro de Sapucaia, utilizando a pesquisa de ACF pelo teste do cartão ICT, sendo diagnosticados $22(6,5 \%)$ antígeno-positivos. Todos os indivíduos positivos foram submetidos à pesquisa de microfilária (Mf) circulante por meio da FSNM, dos quais apenas um (4,5\%) apresentou microfilária circulante. Ante esses achados, o autor chamou a atenção para a importância de se expandir o TM e confirmar a elevada sensibilidade do ACF na população pediátrica, uma vez que os indivíduos desse grupo podem apresentar baixíssimas microfilaremias ou serem amicrofilarêmicos.

Passados 27 anos desde os primeiros atendimentos dos casos de filariose no ambulatório do Subprograma de Filariose do CPqAM (Dreyer, 1987), Souza e colaboradores (2015) descreveram o perfil epidemiológico dos pacientes atendidos no ambulatório do então SRNF/CPqAM no período de 2002 a 2008. Dos 1.109 pacientes atendidos no ambulatório, 947 (85,4\%) eram oriundos da RMR. Na análise estratificada por município, os autores verificaram que Olinda apresentou a menor frequência de indivíduos infectados (11,6\%) contra 43,9\% e 37,8\% para Jaboatão dos Guararapes e Recife, respectivamente. Possivelmente esse resultado seja decorrente dos intensos trabalhos desenvolvidos pelos vários pesquisadores ao longo das décadas de 1980 e 1990. 
Durante o período de 2005 a 2014, no município de Olinda, foram incluídos 17 bairros no TM, sendo administradas cerca de 400.000 doses de DEC à população neles residente (SESO, 2016). Desses 17 bairros, 9 já haviam recebido cinco ou mais doses anuais até 2013. Esses apresentaram ao longo dos anos um percentual de cobertura superior a $65 \%$ e índices de positividade de microfilaremia menores que $1 \%$ no monitoramento dos sítios sentinelas. Tais níveis são considerados adequados segundo os critérios da OMS para a realização do inquérito de avaliação da transmissão TAS, ou Transmission assessment survey (OMS, 2015).

Em 2014, seguindo recomendações da OMS, foi realizado o TAS 1 no município, no qual foram incluídos nove bairros para avaliação da transmissão, divididos em duas áreas de avaliação: OLINDA 1 (Alto do Sol Nascente, Águas Compridas, Alto da Bondade, Alto da Conquista, Passarinho e Caixa D’água) e OLINDA 2 (Peixinhos, Sítio Novo e Salgadinho). Foram incluídas no inquérito todas as escolas desses bairros, sendo examinadas, com o teste do cartão ICT, crianças matriculadas no $1^{\circ}$ e no $2^{\circ}$ ano do ensino fundamental. Como a prevalência de antigenemia com o ICT foi maior que $2 \%$ nas duas unidades de avaliação, o tratamento foi interrompido nessas áreas por dois anos até uma nova avaliação em 2016 quando acontecerá o TAS 2. O TAS 3 ocorrerá em 2019 e o último, TAS 4, será realizado no município em 2021 (SESO, 2016).

O conhecimento gerado ao longo dos 29 anos nos campos do conhecimento clínico, diagnóstico e epidemiologia do agravo FL em OlindaPE vem colaborar sobremaneira com o PNEFL. O município atingiu metas superiores às recomendadas pela OMS para a cobertura do TM, que deve envolver, no mínimo, $65 \%$ da população-alvo a ser medicada. Dessa forma, o momento atual consiste em verificar se essas medidas foram eficazes para a quebra da transmissão. Em 2014, foi implementado o TAS, Coordenado pelas Secretarias Municipal e Estadual de Saúde de Pernambuco em parceria com o Ministério da Saúde do Brasil, Organização Pan-Americana da Saúde e SRNF com um período de dois anos para consolidar os resultados. Portanto, não constam deste trabalho retrospectivo os resultados dos TAS 2, 3 e 4, os quais serão incluídos em um trabalho à parte. Assim, o presente estudo pretende contribuir para a construção do dossiê do Brasil, especificamente no capítulo que trata do histórico das ações de controle realizadas em Olinda-PE, objetivando a eliminação do agravo FL no município. Cabe destacar ainda que as ações direcionadas para a quebra da transmissão contribuem para o não surgimento das formas debilitantes e estigmatizantes da infecção filarial, o que produz impacto direto na melhoria da qualidade de vida da população. 


\section{REFERÊNCIAS}

1. Braga C, Ximenes RAA, Albuquerque MFPM, Souza WV, Miranda J, Brayner F, Alves L, Silva L, Dourado I. Avaliação de indicador sócio-ambiental utilizado no rastreamento de áreas de transmissão de filariose linfática em espaços urbanos. Cad Saúde Públ 17: 1211-1218, 2001.

2. Braga C, Dourado I, Ximenes R, Miranda J, Neal A. Bancroftian filariasis in an endemic area of Brazil: diferences between genders during puberty. Rev Soc Bras Med Trop 38: 224-228, 2005.

3. Brandão E, Oliveira P, Silva M, Quaresma J, Barbosa F, Marcondes M, Silva S, Braga C, Aguiar AS, Rocha A. Freqüência da infecção por Wuchereria bancrofti em crianças em idade escolar, em dois bairros de Olinda-PE, pela técnica do cartão ICT. In: Resumos do XX Congresso Brasileiro de Parasitologia, Recife, PE, 2007. p.56.

4. Cabral SNS. Perfil epidemiológico e antigênico da população não aderente ao tratamento em massa para filariose linfática no município de Olinda-PE. 52p. Dissertação (Mestrado Acadêmico em Saúde Pública), Centro de Pesquisas Aggeu Magalhães, Fundação Oswaldo Cruz, Recife, 2012.

5. Coutinho A, Medeiros Z, Dreyer G. História da filariose linfática em Pernambuco. 1. Aspectos epidemiológicos e de controle. Rev Soc Bras Med Trop 29: 607-612, 1996.

6. Dreyer G. Filariasis programme - Recife, Brasil. Mem Inst Oswaldo Cruz 82: 359-360, 1987.

7. Dreyer G, Rangel A, Estudo epidemiológico da filariose bancroftiana no município de OlindaPE. In: Resumos do I Simpósio Nacional de Filariose, Recife, Brasil.1987.

8. Fontes G, Leite AB, Lima ARV, Freitas H, Ehrenberg JP, Rocha EMM. Lymphatic filariasis in Brazil: epidemiological situation and outlook for elimination. Parasit Vectors 5: 272-283, 2012.

9. Freitas H, Vieira JB, Braun R, Medeiros Z, Rocha EMM, Aguiar-Santos AM, Fraiha H, Rocha A. Workshop para a avaliação da situação epidemiológica da filariose linfática no município de Belém, Pará, Norte do Brasil. Rev Soc Bras Med Trop 41: 212-216, 2008.

10. FUNASA. Fundação Nacional de Saúde. Centro Nacional de Epidemiologia. Programa de eliminação da filariose bancroftiana nas Américas. Boletim Epidemiológico 1: 12, 1997.

11. IBGE. Instituto Brasileiro de Geografia e Estatística. Censo demográfico 2010: Características populacionais e domiciliares. Rio de Janeiro, 2010. http://www.ibge.gov.br acesso em: 15 de out. 2015.

12. Maciel MA, Marzochi, KB, Silva EC, Rocha A, Furtado AF. Estudo comparativo de áreas endêmicas de filariose bancroftiana na Região Metropolitana do Recife, Brasil. Cad Saúde Públ 10: S301-S309, 1994.

13. Marcondes MMC. Conhecimentos e Práticas em Portadores de Morbidades Filarial Linfática no Municipio de Olinda-PE, Brasil. 132 p. Dissertação (Mestrado Profissional em Saúde Pública), Centro de Pesquisa Aggeu Magalhães, Fundação Oswaldo Cruz, Recife, 2010.

14. Medeiros Z, Dreyer G, Andrade L, Pires ML, Mendes J, Pimentel R. Wuchereria bancrofti microfilarial density of autochthonous cases and natural Culex infectivity rates in Northeast Brazil. J Trop Med Hyg 95: 214-217, 1992.

15. Miranda J, Maciel A, Souza RMC, Furtado AF, Malagueno E. Perfil protéico e reconhecimento antigênico de extratos de larvas infectantes (L3) de Wuchereria bancrofti. Rev Soc Bras Med Trop 38: 27-32, 2005.

16. Molyneux DH, Neira M, Liese B, Heymann. Elimination of lymphatic filariasis as a public health problem. Lymphatic filariasis: setting the scene for elimination. Trans Roy Soc Trop Med Hyg 94: 589-591, 2000.

17. MS. Ministério da Saúde. Fundação Nacional de Saúde: Campanha de saúde pública, departamento de erradicação e controle de endemias. In: O controle das endemias no Brasil de 1979 a 1984. Brasília, 1: 130-133, 1985. 
18. MS. Ministério da Saúde. Plano integrado de ações estratégicas de eliminação da hanseníase, filariose, esquistossomose e oncocercose como problema de saúde pública, tracoma como causa de cegueira e controle das geohelmintíases: Plano de ação 2011-2015. Brasil. Secretaria de Vigilância em Saúde. Departamento de Vigilância de Doenças Transmissíveis. Brasília, 2012.

19. OMS. Organização Mundial de Saúde. Programa mundial para eliminação da filariose linfática: Treinamento em monitoramento e avaliação epidemiológica da intervenção com tratamentos coletivos para eliminação da filariose linfática. Genebra, $2015.89 \mathrm{p}$.

20. OPAS. Organização Pan Americana de Saúde. Condições de saúde e suas tendências. In: Saúde nas Américas. Washington, 1: 62-217, 2007.

21. Ottesen EA, Duke BOL, Karam M, Behbehani K. Strategies and tools for the control/ elimination of lymphatic filariasis. Bull World Health $\operatorname{Org}$ 75: 491-503, 1997.

22. PAHO. Pan American Health Organization. Strengthening surveillance to achieve and sustain elimination of transmission of Lymphatic Filariasis and tackle other neglected infectious diseases in the region of the Americas. In: $16^{\text {th }}$ Regional Lymphatic Filasiasis Elimination Program Managers' Meeting. Recife, Brazil: PAHO; 2015.

23. Rachou R. Conceito e programa de profilaxia da filariose bancroftiana no Brasil. Rev Bras Malariol e Doenças Trop 12: 11-39, 1960.

24. Rocha A, Braun R, Quaresma J, Costa J, Aguiar-Santos AM, Freitas H, Bonfim C, Andrade $\mathrm{LD}$, Oliveira C, Ault S, Medeiros Z. Inquérito antigênico da filariose bancroftiana em Belém do Pará: Verificação da ausência de infecção por Wuchereria bancrofti. Rev Soc Bras Med Trop 38: S475-S476, 2005.

25. Rocha A, Marcondes M, Nunes JRV Miranda T, Veiga J, Araújo P, Tenório W, Santos AA. Programa de controle e eliminação da filariose linfática: uma parceria da Secretaria de Saúde de Olinda, PE, Brasil, com o Serviço de Referência Nacional em Filariose. Rev Patol Trop 39: 233-249, 2010.

26. Secretaria Estadual de Saúde de Olinda (SESO). 2016. Dados não publicados.

27. Silva JSF. Cinética de Microfilaremia, Antigenemia e Anticorpos antifilariais em Indivíduos infectados por Wuchereria bancrofti, tratados com Doses Únicas anuais de Dietilcarbamazina em uma Área Endêmica no município de Olinda, PE. 48p. Dissertação (Mestrado Acadêmico em Saúde Pública), Centro de Pesquisa Aggeu Magalhães, Fundação Oswaldo Cruz, Recife, 2014.

28. Souza PFA, Brandão E, Aguiar-Santos AM, Medeiros Z, Silva RLF, Oliveira P, Araújo PSR, Teixeira MJ, Rocha A. Perfil epidemiológico de paciente atendidos no ambulatório do Serviço de Referência Nacional em Filarioses do Centro de Pesquisas Aggeu Magalhães, Recife-PE. Rev Patol Trop 43: 427-435, 2015.

29. WHO. World Health Organization. Global Programme to Eliminate Lymphatic Filariasis: Progress report, 2014. Weekly Epidemiological Record, Geneva, 38: 489-504, 2015. 